Cahiers $d u$ MONDE RUSSE

\section{Cahiers du monde russe}

Russie - Empire russe - Union soviétique et États indépendants

$55 / 3-4 \mid 2014$

Varia

\title{
Violeta Davoliūtè, The Making and Breaking of Soviet Lithuania, Memory and modernity in the wake of war
}

\section{Alain Blum}

\section{(2) OpenEdition}

1 Journals

\section{Édition électronique}

URL : http://journals.openedition.org/monderusse/8118

DOI : $10.4000 /$ monderusse. 8118

ISSN : $1777-5388$

Éditeur

Éditions de l'EHESS

\section{Édition imprimée}

Date de publication : 1 juillet 2014

Pagination : 482-486

ISBN : 978-2-7132-2441-6

ISSN : $1252-6576$

\section{Référence électronique}

Alain Blum, «Violeta Davoliūtè, The Making and Breaking of Soviet Lithuania, Memory and modernity in the wake of war », Cahiers du monde russe [En ligne], 55/3-4 | 2014, mis en ligne le 14 avril 2015, Consulté le 24 septembre 2020. URL : http://journals.openedition.org/monderusse/8118; DOI https://doi.org/10.4000/monderusse.8118

Ce document a été généré automatiquement le 24 septembre 2020.

(c) École des hautes études en sciences sociales 


\title{
Violeta Davoliūtè, The Making and Breaking of Soviet Lithuania, Memory and modernity in the wake of war
}

\author{
Alain Blum
}

\section{RÉFÉRENCE}

Violeta DAVOLIUTE, The Making and Breaking of Soviet Lithuania, Memory and modernity in the wake of war, London - New York : Routledge, 2013, 211 p.

1 Une histoire complexe des sociétés des territoires qui constituèrent l'URSS reste à faire, en particulier celles des sociétés des territoires annexés après 1939 qui connurent une soviétisation accélérée après la Seconde Guerre mondiale; une histoire qui ne se résumerait pas à une hagiographie de la résistance de tel ou tel peuple à une soviétisation qui divise la société en un groupe minoritaire, tenu par le centre et détenteur des commandes, et un groupe majoritaire, contraint, mais mettant tout en œuvre pour s'opposer au premier. Dans l'interaction qui s'établit entre les nouvelles formes de pouvoir et les acteurs sociaux, on ne peut à l'évidence réduire les actions sociales à une opposition entre deux extrêmes, résistance ou collaboration, intégration ou exclusion. Les formes d'intégration et de transformation sont bien plus complexes et nuancées.

2 Il est vrai que certains travaux se sont déjà engagés dans cette écriture, celui d'Elena Zubkova ${ }^{1}$, bien entendu, mais aussi ceux d'olaf Mertelsmann ${ }^{2}$, ou encore la thèse en cours de Juliette Denis. L'ouvrage de Violeta Davoliūte contribue clairement à cette nouvelle historiographie qui ne résume pas le monde à deux clans opposés ni la soviétisation à un simple contrôle, subi, des populations de ces territoires. À la différence des travaux évoqués ci-dessus, les sources de son étude ne sont pas tant les 
archives qu'un grand nombre d'entretiens qu'elle a menés avec les élites intellectuelles lituaniennes, aujourd'hui présentes et souvent actives en Lituanie indépendante mais qui furent tout autant actrices de la Lituanie soviétique. V. Davoliūtė se fonde aussi sur une solide connaissance de l'historiographie la plus récente sur ce pays, maîtrisant aussi bien la littérature anglo-saxonne, que lituanienne. Elle cherche à comprendre les transformations brutales qui affectèrent le pays, conséquences tout autant de l'occupation soviétique que des bouleversements induits par la Seconde Guerre mondiale.

Dans un premier constat, V. Davoliūtè souligne l'impossibilité de comprendre l'histoire de la Lituanie soviétique si l'on ne tient pas compte du bouleversement urbain (War, the city and the country) auquel le pays a été confronté. Peuplées en grande partie de Polonais et de Juifs avant la guerre (en particulier Vilnius), les villes se sont vidées de ces deux populations, les premiers ont rejoint la Pologne, les seconds ont été exterminés durant la Seconde Guerre mondiale. Cette hémorragie, suivie de la collectivisation des terres et des mouvements insurgés luttant contre la présence des Soviétiques, va provoquer des flux considérables de population vers les grandes villes, qui se peuplent alors très rapidement d'une population rurale. Pour l'auteur, c'est bien dans la ville que se déroule l'histoire de la Lituanie soviétique, mais une ville bouleversée, dans laquelle l'habitat urbain anciennement largement peuplé de Polonais et de Juifs, est désormais investi par des paysans lituaniens.

4 C'est ainsi toute une génération qui va bénéficier d'une ascension sociale considérable. À l'instar de Justinas Marcinkevičius, l'écrivain intellectuel emblématique de la Lituanie soviétique comme de la Lituanie contemporaine, ou encore d'Algimantas Baltakis, la génération des années 1930 profitera de cette ascension tout en contribuant à la renaissance d'une certaine culture lituanienne. Dans le chapitre Soviet Lithuanian renaissance, l'auteur montre bien les formes de compromis permanents qui s'établissent entre le parti et ces nouveaux écrivains. Ces formes de compromis s'expriment aussi dans une réelle ouverture, par ailleurs croissante, de Sniečkus, le premier secrétaire du parti de Lituanie. Au demeurant, la personnalité de celui-ci semble jouer un rôle important dans cette histoire, entre contrôle et appui sur cette jeune génération, à laquelle il offre une certaine ouverture, que 1956 remet moins en cause que dans d'autres États du bloc de l'Est.

5 Viennent alors les prémices d'une forme de modernité soviétique, dans laquelle les trois Républiques baltes, et plus particulièrement étudiée ici la Lituanie, jouent un rôle crucial d'Ouest soviétique ${ }^{3}$, territoire intermédiaire où s'expriment une plus grande ouverture et, de ce fait aussi, une plus grande espérance dans une forme d'internationalisation d'une culture née de l'après-guerre. Violeta Davoliūtè montre comment cette modernité s'exprime parmi les élites qui attendent des formes de reconnaissance internationale, recevant des écrivains occidentaux ou voyageant elles-mêmes. Mais elles se heurtent vite à des formes de rejet, à l'expression d'un jugement de l'Ouest quelque peu compatissant, voire méprisant. À cet égard, la description qui est faite de la visite de Jean-Paul Sartre et Simone de Beauvoir en Lituanie en 1965, puis la rencontre à Paris entre le premier et Justinas Marcinkevičius constituent un des moments étonnants de ce livre. Entre découverte et désillusion, les élites lituaniennes vont alors se tourner vers une autre expression à connotation nationale, tout en s'inscrivant dans la dynamique assez générale en URSS au même moment du rustic turn: "Broad cultural reaction to the failure of Soviet modernity, 
which gathered speed through the 1970s and was expressed as a return to the rural, pre-modern roots of identity. It began as a vague sense of nostalgia for the lost way of life and grew into a politically explosive discourse of collective trauma " (p.124). Ce tournant, Violeta Davoliūtè en fait un moment essentiel qui relie cette période à la création de Sajūdis dans les années 1980, puis au rassemblement étonnant autour de la déportation de l'expression d'une identité lituanienne, qui subvertit le soviétisme, de l'ensemble des élites et de la population de la Lituanie indépendante. Certes, le rustic turn fait en partie écho à la prose paysanne des années 1970 en Russie, mais il prend une dimension particulière en Lituanie, permettant des formes d'autonomisation nationale. Il se caractérise par le développement d'expressions folkloriques de ce que serait une culture nationale, mises en scènes aussi bien dans des ensembles de danse que dans le cinéma ou la photographie, qui valorise "un monde que nous avons perdu ». Une très belle photo d'Antanas Sutkus, présente dans ce livre, exprime avec grande acuité cette renaissance du village lituanien dans les représentations qui sont alors publiées.

6 Derrière ce rustic turn se préparent d'étranges rapprochements, qui vont conduire à faire des déportations staliniennes le destin partagé de toute la population de Lituanie. Reprenant dans cet ouvrage les thèses développées dans un article approfondi sur la question ${ }^{4}$, Violeta Davoliūte montre comment la rencontre improbable entre Justinas Marcinkevičius et Dalia Grinkevičiūtè va permettre cette synthèse. Le premier reste, après l'indépendance recouvrée de la Lituanie, un des écrivains principaux et respectés de la Lituanie. La seconde, qui a vécu l'expérience la plus traumatique de toutes les déportations de Lituanie, celle de la déportation sur la mer de Laptev ${ }^{5}$, a été confrontée à plusieurs formes d'exclusion après son retour. Or, c'est grâce au premier que les Mémoires de la seconde sont publiées en Lituanie en $1997^{6}$ et fondent la mémoire publique partagée de la déportation des Lituaniens. Les élites intellectuelles de la période soviétique sont alors porteuses d'une continuité avec la période de l'indépendance. Cette continuité s'exprime très bien à travers cette synthèse issue de la rencontre entre l'emblème, qui conserve son prestige après 1991, d'une littérature soviétique lituanisée et l'emblème, réprimé, déporté dans un des lieux les plus tragiques de l'histoire des déportations staliniennes, de la population de Lituanie.

7 L'ouvrage de Violeta Davoliūté réussit ainsi à proposer une histoire cohérente et en même temps complexe, qui relie les années d'avant-guerre à la période contemporaine avec un sens tout à fait convaincant de la continuité. Il éclaire la position des élites intellectuelles soviétiques dans la Lituanie indépendante, tout en l'articulant avec les profondes transformations subies par ce pays. Il ne présente ni une histoire soviétique ni une histoire lituanienne, mais bien une histoire de relations, de tensions, de compromis qui traversent la période.

Même partielle, ce qui tend à fragiliser parfois la démonstration, cette histoire reste convaincante. Cependant, il manque un regard plus précis sur les formes de pression, d'intimidation des services policiers sur les élites, pour mieux comprendre les compromis auxquels ces dernières devaient consentir. Il manque aussi sans doute une analyse approfondie des éléments qui faisaient passer ces élites d'un côté ou de l'autre de l'opposition au système, ou qui les conduisaient à mettre à l'épreuve ce qu'elles pouvaient exprimer. Ici, les archives policières ne sont que trop peu utilisées : il aurait été vraiment intéressant de confronter le regard des élites intellectuelles, en particulier quand elles font le récit de cette histoire, à ceux de l'appareil policier et de l'appareil 
politique. Dès le milieu des années 1960 et à différents niveaux, les organes de surveillance ainsi que le parti sont particulièrement attentifs à ces élites intellectuelles. C'est le moment où la surveillance change de cible, abandonnant le milieu rural pour le milieu urbain, et passant des paysans aux élites intellectuelles ou aux étudiants. Juste après la guerre, les milieux ruraux, qui avaient beaucoup contribué à lutter contre les Soviétiques, avaient subi une répression massive entre 1945 et 1953. S'ils sont surveillés lors des retours de déportation qui s'étendent jusqu'au milieu des années 1960, rapidement la surveillance se tourne vers les milieux urbains. De telles investigations auraient sans doute permis de mieux comprendre les regards croisés, et donc la relation entre ces élites et le pouvoir.

Quelques conclusions apparaissent un peu rapides, en particulier lorsque l'auteur s'éloigne des élites intellectuelles pour traiter des retours de déportation. On aurait souhaité mieux comprendre les conséquences sur le monde rural de cet écart entre deux mondes, celui d'avant la déportation et celui du retour. Violeta Davoliūté souligne à quel point ces déportés sont perdus lors du retour, tant l'abîme est profond entre la Lituanie qu'ils ont quittée en 1948-1949 et celle qu'ils retrouvent au début des années 1960. Mais n'avaient-ils pas, à travers les diverses modalités d'adaptation en déportation, acquis une certaine expérience qui leur eût permis de mieux comprendre ce décalage?

Enfin, il n'est pas certain que les conclusions les plus fortes de cet ouvrage puissent être étendues à l'ensemble de la population. Elles reposent essentiellement sur une histoire des élites et sur une histoire culturelle plus que sociale, même si cette dernière dimension est bien présente. L'auteur n'a d'ailleurs pas cette ambition, mais glisse parfois un peu rapidement vers des formes de généralisation qui ne sont pas vraiment démontrées.

Il n'en reste pas moins qu'il s'agit là d'un ouvrage important dans l'écriture de ces histoires nationales au sein de l'URSS, histoire qui, à défaut d'être globale, se focalise de façon très heureuse sur un pan de la société, sur des mondes particuliers qui ne se réduisent pas à des collaborateurs et des résistants, ou à des occupants et des occupés. L'approche très subtile et fine que présente Violeta Davoliūté constitue une nouvelle étape dans l'écriture de cette histoire.

\section{NOTES}

1. Elena Zubkova, Pribaltika i Kreml', 1940-1953 [Les territoires baltes et le Kremlin, 1940-1953], M. : ROSSPEN, 2008.

2. Olaf Mertelsmann, The Sovietization of the Baltic States, 1940-1956, Tartu : KLEIO Ajalookirjanduse Sihtasutus, 2003 ; Olaf Mertelsmann, Everyday Life in Stalinist Estonia, Frankfurt am Main - New York : Peter Lang, 2012.

3. Idée aussi fortement présente chez Zubkova, Pribaltika i Kreml', par exemple.

4. Violeta Davoliūtè, "We Are All Deportees." The Trauma of Displacement and the Consolidation of National Identity during the Popular Movement in Lithuania ", in Violeta 
Davoliūtè, Tomas Balkelis, éds., Maps of Memory: Trauma, Identity and Exile in Deportation Memoirs from the Baltic States, Vilnius, 2012.

5. Voir à ce propos quelques autres témoignages dans museum.gulagmemories.eu, en particulier celui d'Irena Ašmontaitè-Giedrienè (http://museum.gulagmemories.eu/fr/salle/irenaasmontaite-giedriene

6. Dalia Grinkevičiūtè, Lietuviai prie LaptevŲ jūros: Atsiminimai, miniatiūros, Vilnius : A. Šulskytè, 1997.

\section{AUTEURS}

\section{ALAIN BLUM}

CNRS - EHESS Paris, Centre d'études franco-russes de Moscou 